\title{
Vibrio vulnificus septicemia and necrotizing fasciitis in the patients with liver cirrhosis and diabetes mellitus
}

\author{
Hiroyuki Ito ${ }^{1^{*}}$, Akane Shibayama $^{1}$, Mariko Abe $^{1}$, Shinichi Antoku $^{1}$, Hiroko Nawata ${ }^{1}$, \\ Miyuki Isonishi ${ }^{2}$, Masaki Fujita ${ }^{3}$, Shojiro Kato ${ }^{4}$ \\ ${ }^{1}$ Department of Diabetes, Metabolism and Kidney Disease, Edogawa Hospital, Tokyo, Japan; \\ *Corresponding Author: ito@edogawa.or.jp \\ ${ }^{2}$ Laboratory Department, Edogawa Hospital, Tokyo, Japan \\ ${ }^{3}$ Department of Cardiology, Edogawa Hospital, Tokyo, Japan \\ ${ }^{4}$ Department of Orthopedics, Edogawa Hospital, Tokyo, Japan
}

Received 2 December 2011; revised 5 January 2012; accepted 14 January 2012

\begin{abstract}
Vibrio vulnificus (V. vulnificus) infection is a rare disease in Japan but the leading cause of death related to raw seafood consumption. We hereby reported a successfully treated case of $V$. vulnificus septicemia, severe necrotizing fasciitis, disseminated intravascular coagulation and multiple organ failure after raw perch consumption with underlying alcoholic liver cirrhosis and diabetes mellitus. It is the first report of a case of $V$. vulnificus infection caused by eating raw perch, whereas $V$. vulnificus infection should be suspected in all of middle-aged to elderly men with underlying immunosuppressive diseases, who have recent consumption of raw seafood or contact with seawater, especially in the summer. The levels of HbA1c and glycoalbumin were not high in the present case, however, obvious hyperglycemia was found even after the infection had completely healed. On reviewing 166 case of $V$. vulnificus infection in Japan including ours, the complication of diabetes mellitus, one of immunocompromised condition, was found only in $11 \%$, although it had been reported that individuals strongly suspected of having diabetes were $17.2 \%$ among the Japanese male population aged from $\mathbf{4 0}$ to $\mathbf{7 4}$ years. Because diabetes mellitus might be underdiagnosed in the previous reports, intensive examinations are considered to be necessary in order to correctly diagnose diabetes mellitus in patients with severe $V$. vulnificus infection.
\end{abstract}

Keywords: Vibrio vulnificus; Primary Septicemia; Necrotizing Fasciitis; Liver Cirrhosis; Diabetes
Mellitus

\section{INTRODUCTION}

Vibrio vulnificus ( $V$. vulnificus) is a marine gramnegative bacterium that is endemic in warm coastal waters. $V$. vulnificus infection results in 3 clinical syndromes: primary septicemia, wound infection and gastrointestinal illness [1]. Individual who are most susceptible to $V$. vulnificus infection usually suffer from a chronic disease that affects either liver function or the immune system [2]. $V$. vulnificus infection causes fulminant and invasive disease and has a high mortality rate. There have been numerous reports of $V$. vulnificus infection in Japan since 1978 [3]. Here, a successfully treated case of $V$. vulnificus septicemia complicated with shock, necrotizing fasciitis, disseminated intravascular coagulation (DIC), and multiple organ failure (MOF) is presented. The literature, including 166 reported case of this infection in Japan, is also reviewed.

\section{CASE REPORT}

A 60-year-old man with swelling of both lower extremities was admitted to our hospital on July, 2007. The medical history of the patient was unclear because he had not undergone any prior physical checkup. Five days earlier, he had suffered from discomfort, subjective fever, and pain in both lower extremities. He was an inhabitant of Tokyo; he had consumed sliced law perch a week prior to the onset of clinical symptoms, but there was no history of contact with seawater at that time. He confessed alcohol abuse and refused any prior episode of trauma.

On admission, he was afebrile, his blood pressure was $101 / 70 \mathrm{mmHg}$, and his heart rate was 92 beats/min. His 
lower extremities showed free movement but were swollen and painful with purpuric macules, hemorrhagic bullae and epidermolysis (Figure 1). The white blood cell count was $9.2 \times 10^{9} / \mathrm{L}$ (neutrophil $91 \%$ ); platelet count, $35 \times 10^{9} / \mathrm{L}$; and hemoglobin level $115 \mathrm{~g} / \mathrm{L}$. Other laboratory findings were as follows: serum albumin, $16 \mathrm{~g} / \mathrm{L}$; cholinesterase, $74 \mathrm{IU} / \mathrm{L}$; total cholesterol, $1.97 \mathrm{mmol} / \mathrm{L}$; total bilirubin, $44.5 \mu \mathrm{mol} / \mathrm{L}$; aspartate aminotransferase, $85 \mathrm{IU} / \mathrm{L}$; alanine aminotransferase, $33 \mathrm{IU} / \mathrm{L}$; alkaline phosphatase, $410 \mathrm{IU} / \mathrm{L} ; \gamma$ glutamyl transpeptidase, 263 $\mathrm{IU} / \mathrm{L}$; lactate dehydrogenase, $306 \mathrm{IU} / \mathrm{L}$; creatine kinase, $684 \mathrm{IU} / \mathrm{L}$; blood urea nitrogen, $9.64 \mathrm{mmol} / \mathrm{L}$; serum creatinine, $206.86 \mathrm{mmol} / \mathrm{L}$; blood sugar $15.0 \mathrm{mmol} / \mathrm{L}$; C-reactive protein, $8.30 \mathrm{mg} / \mathrm{dL}$; and antistreptolysin- $\mathrm{O}$, 237 U/L. Hemoglobin A1c (HbA1c) and glycated albumin (GA) was $4.9 \%$ and $14.9 \%$, respectively. The HbAlc level was calibrated by the Japan Diabetes Society (JDS) standard calibrators. The value for HbAlc (\%) is estimated as a National Glycohemoglobin Standardization Program (NGSP) equivalent value (\%) calculated by the formula: HbA1c (JDS) + 0.4 [4]. The antistreptokinase level was 640x higher than the normal level. Either hepatitis B surface antigen or hepatitis C virus antibody was negative. Arterial gas analysis, performed when the patients was breathing room air, showed the following findings: $\mathrm{pH}, 7.397 ; \mathrm{PaCO}_{2} 17.9 \mathrm{mmHg} ; \mathrm{PaO}$ 2, 93.9 $\mathrm{mmHg}$; and bicarbonate, $10.8 \mathrm{mmol} / \mathrm{L}$. The prothrombin time was $50.5 \%$; prothrombin time international normalized ratio, 1.72; activated partial thromboplastin time, $39.5 \mathrm{~s}$; fibrinogen level, $8.96 \mu \mathrm{mol} / \mathrm{L}$; and fibrin degeneration product level, $56 \mu \mathrm{g} / \mathrm{mL}$. A chest radiograph showed cardiomegaly with a cardiothoracic ratio of $54.8 \%$, and bilateral pleural effusion. A computerized tomography scan of the lower extremities showed honeycomb patterns in the fascia, fat and muscle (Figure 2). Necrotizing fasciitis was diagnosed on the basis of necrosis of subcutaneous tissue and fascia, with variable involvement of the skin and muscle.

The patient was immediately administered pazufloxacin $(500 \mathrm{mg})$ and clindamycin $(600 \mathrm{mg})$ at $12 \mathrm{~h}$ intervals. At $12 \mathrm{~h}$ after admission, the patients condition suddenly worsened, and he developed septic shock. A repeat chest radiograph showed progressive bilateral pulmonary congestion and pleural effusion. Echocardiography of the left ventricle showed diffuse hypokinesis and an ejection fraction of $44 \%$. The patient was diagnosed with fulminant DIC and MOF. Dopamine and norepinephrine were administered, and endotoxin absorption therapy was performed by means of hemodialysis. The patient was administered a daily dose of gabexate $(2000 \mathrm{mg})$ and heparin $(10,000$ units) combined with antithrombin III (1500 units) as emergent therapy for DIC. Additionally, insulin infusion and sliding scale were administered at a dosage of 70 units/day in order to achieve glycemic con-
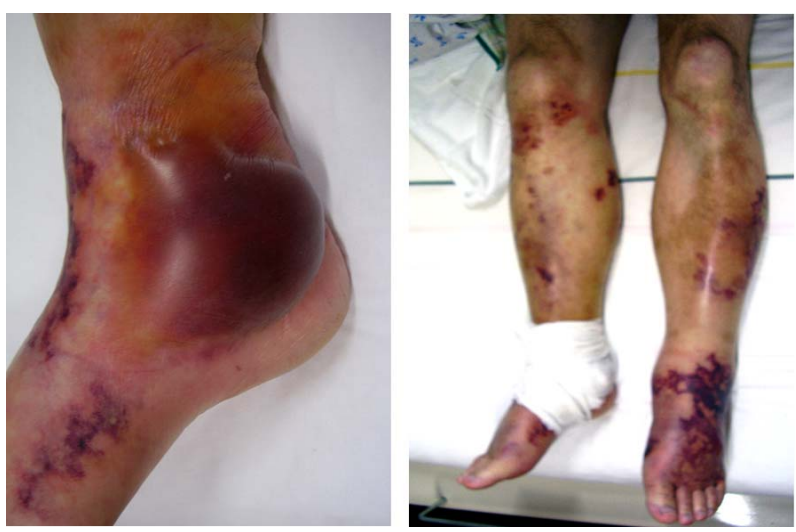

Figure 1. Skin lesions with purpuric macules, hemorrhagic bullae, and epidermolysis on both the lower extremities up to the knees at the time of admission; Vibrio vulnificus was not detected in the material aspirated from a local lesion. The photograph has been reproduced after receiving the patient's consent.
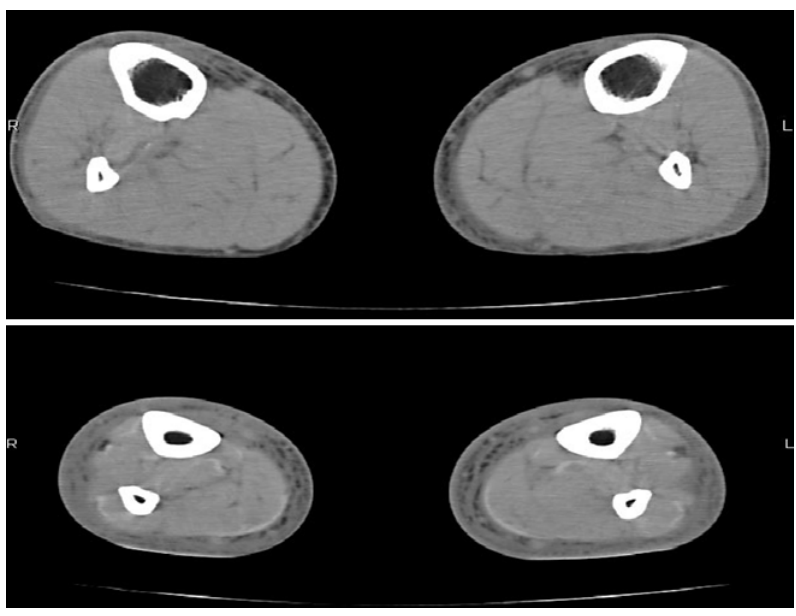

Figure 2. Computerized tomography scan of the extremities at the time of admission showed honeycomb patterns in the fascia, fat and muscle.

trol, i.e., a blood sugar level of less than $11.1 \mathrm{mmol} / \mathrm{L}$. Blood culture examination performed at the time of admission was positive for a gram-negative bacillus, which was identified as $V$. vulnificus on the fifth hospital day; however, cultures of fluid obtained from the hemorrhagic bulla and the ankle joint were negative. Subsequently, the patients was administered cefzopram $(1 \mathrm{~g})$ every $12 \mathrm{~h}$ because $V$. vulnificus is sensitive to this antibiotic. On the 30th hospital day, insulin was replaced with mitiglinide, which is also known as a rapid-acting insulin secretagogue. The sizes $f$ skin lesions were subsequently reduced by the management with closed wet dressings.

When the patients achieved cardiovascular stability, the indocyanine green retention test and $75 \mathrm{~g}$ oral glucose tolerance test (OGTT) were performed to confirm liver cirrhosis and diabetes mellitus, respectively. Alcoholic liver cirrhosis was diagnosed on the basis of indo- 
cyanine green retention of $54.8 \%$ at $15 \mathrm{~min}$ and the findings according to abdominal ultrasonography. Diabetes mellitus was diagnosed on the basis of the OGTT findings of blood sugar level of $11.7 \mathrm{mmol} / \mathrm{L}$ at $120 \mathrm{~min}$ and a random blood sugar level of greater than $11.1 \mathrm{mmol} / \mathrm{L}$ since admission. On the 120th hospital day, his blood urea nitrogen level improved to $4.28 \mathrm{mmol} / \mathrm{L}$ and creatinine level to $43.47 \mu \mathrm{mol} / \mathrm{L}$. Early diabetic nephropathy was diagnosed on the basis of a urinary albumin excretion rate of $32.2 \mathrm{mg} / \mathrm{g} / \mathrm{creatinine}$. Fundoscopic examination showed no diabetic retinopathy. Diabetic neuropathy was difficult to evaluate because of the skin lesions on both the lower extremities. At this stage, the urinary C-peptide immunoreactivity was $56.5 \mu \mathrm{g} /$ day when on mitiglinide treatment, HbAlc, 5.2\%; and GA $16.6 \%$, and the hemoglobin level had improved to 131 $\mathrm{g} / \mathrm{L}$ and albumin level to $46 \mathrm{~g} / \mathrm{L}$. He was discharged on the 154th hospital day, and 7 months later, the skin lesions had almost healed.

\section{DISCUSSIONS}

Necrotizing fasciitis is a rare infection of the deep layers of the skin and subcutaneous tissue; it is easily spreads to the fascia and is commonly known as a fresheating disease. Most case of necrotizing fasciitis are caused by group A (beta) hemolytic Streptococcus pyogenes; and the other causative organism are Staphylococcus aureus, V. vulnificus, Clostridium perfringens, and Bacteroides fragilis [5,6].

V. vulnificus is a halophilic, rod-shaped, gram-negative bacillus of the family Vibrionaceae. It is commonly found in warm coastal waters and is isolated in high concentrations from shellfish, especially oysters [7]. Most cases of $V$. vulnificus infection are recognized as primary bacteremia manifesting as skin and soft tissue infection, occurring mainly in men over 40 years of age with a history of excessive alcohol consumption and underlying hepatic disease such as liver cirrhosis or hepatitis.

Our patient was a typical case of this infection: he was a 60-year-old man with alcoholic liver cirrhosis and diabetes mellitus. $V$. vulnificus infection occurs in the summer, as seen in our case. Epidemiological history obtained from the patients revealed no recent contact with seawater or ingestion of oysters; however, he had consumed slice raw perch 1 week prior to the onset of illness. To the best of our knowledge, ours is the first report of a case of $V$. vulnificus infection caused by eating raw perch.

Diabetes mellitus and liver cirrhosis reduce host resistance to bacterial infections owing to the immunocompromised status. HbA1c or GA are common indicators of long-term glycemic control, but both levels of these pa- rameters were almost normal in our patients because of anemia or hypoalbuminemia due to underlying liver cirrhosis and severe infection. To minimize the occurrence of the potentially fatal infection, patients with such underlying diseases should avoid eating undercooked seafood or exposing open wound to seawater and marine products, especially in the summer or during warm weather.

On reviewing the 166 cases of $V$. vulnificus infection in Japan [8-14] including our case (Table 1), the average age of the patients was 60 years old (range, 23 to 81) and approximately $90 \%$ were men. Most of the patients had septic type infection with high mortality rate. Sixty-nine of 166 patients $(42 \%)$ had liver cirrhosis and 18 (11\%) had diabetes mellitus. This complication rate of diabetes mellitus seems too low compared to the prevalence of diabetes mellitus in Japan. The Ministry of Health, Labour and Welfare of Japan reported that individuals strongly suspected of having diabetes were $17.2 \%$ among the male population aged from 40 to 74 years [15]. Although the levels of HbA1c and GA were not high in the present case, obvious hyperglycemia was found even after the infection had completely healed. Anemia and hypoalbuminemia are common in patients with severe $V$. vulnificus infection, especially in those with liver cirrhosis. Because HbA1c or GA is not useful for the diagnosis of diabetes mellitus in patients with anemia or hypoalbuminemia, diabetes mellitus might be underdiagnosed in the previous reports. Intensive examinations are considered to be necessary in order to correctly diagnose diabetes mellitus in patients with severe $V$. vulnificus

Table 1. Review of 166 cases of Vibrio vulnificus infection reported in Japan.

\begin{tabular}{ccc}
\hline Clinical characteristics & Mean \pm SD/Case number & Mortality rate \\
\hline Age (years) & $60.4 \pm 12.2$ & \\
Men & $148(89 \%)$ & \\
Month of onset & & \\
July to September & $115(69 \%)$ & \\
Other & $27(16 \%)$ & \\
Unknown & $24(15 \%)$ & $23(33 \%)$ \\
Underlying disease & & $9(50 \%)$ \\
Liver cirrhosis & $69(42 \%)$ & \\
Diabetes mellitus & $18(11 \%)$ & $101(74 \%)$ \\
Clinical presentation & & $7(29 \%)$ \\
Primary septicemia & $137(83 \%)$ & $0(0 \%)$ \\
Wound infection & $24(13 \%)$ & \\
Gastrointestinal illness & $5(3 \%)$ & \\
\hline
\end{tabular}


infection.

Mortality rates related to necrotizing fasciitis in the case of group A (beta) hemolytic Streptococcus pyogenes range from $20 \%$ to $60 \%$ [16], and from $50 \%$ to $70 \%$ in the case of $V$. vulnificus infection because of the development of primary septicemia [17]. It is difficult to distinguish these infections from histopathological findings, and a history of recent consumption of raw seafood is the only clue to $V$. vulnificus, especially in the patients with an immunocompromised status [11].

Treatment of $V$. vulnificus infection includes antibiotics, skin lesions therapy, and management of shock and DIC. Early suspicion of this infection and aggressive antibiotic treatment are required to avoid fatal outcome. $V$. vulnificus infection should be suspected in all of middle-aged to elderly men with underlying immunosuppressive diseases, who have recent consumption of raw seafood or contact with seawater, especially in the summer.

\section{REFERENCES}

[1] Klontz, K.C., Lieb, S., Schreiber, M., Janowski, H.T., Baldy, L.M. and Gunn, R.A. (1988) Syndromes of Vibrio vulnificus infections. Clinical and epidemiologic features in Florida cases, 1981-1987. Annals of Intern Medicine, 109, 318-323.

[2] Tacket, C.O., Brenner, F. and Blake, P.A. (1984) Clinical features and an epidemiological study of Vibrio vulnificus infections. The Journal of Infectious Diseases, 149, 558561. doi:10.1093/infdis/149.4.558

[3] Miya, H. (1978) Fulminating lactose-positive Vibrio septicemia. Acta Pathologica Japonica, 28, 937-948.

[4] Seino, Y., Nanjo, K., Tajima, N., Kadowaki, T., Kashiwagi, A., et al. (2010) Report of the committee on the classification and diagnostic criteria of diabetes mellitus. Journal of Diabetes Invest, 1, 213-228.

[5] Singh, G., Sinha, S.K., Adhikary, S., Babu, K.S., Ray, P. and Khanna, S.K. (2002) Necrotising infections of soft tissues-A clinical profile. The European Journal of Surgery, 168, 366-371. doi:10.1080/11024150260284897

[6] Elliott, D.C., Kufera, J.A. and Myers, R.A. (1996) Necrotizing soft tissue infections. Risk factors for mortality and strategies for management. Annals of Surgery, 224, 672-683. doi:10.1097/00000658-199611000-00011

[7] Harwood, V.J., Gandhi, J.P. and Wright, A.C. (2004) Methods for isolation and confirmation of Vibrio vulnificus from oysters and environmental sources: A review.
Journal of Microbiological Methods, 59, 301-316. doi:10.1016/j.mimet.2004.08.001

[8] Inoue, Y., Ono, T., Matsui, T., Miyasaka, J., Kinoshita, Y. and Ihn, H. (2008) Epidemiological survey of Vibrio vulnificus infection in Japan between 1999 and 2003. The Journal of Dermatology, 35, 129-139. doi:10.1111/j.1346-8138.2008.00432.x

[9] Kikawa, K., Yamasaki, K., Sujiura, T., Myose, H., Chinen, M., Tsutsumi, K., Iwao, N. and Dohzono, T. (1990) A successfully treated case of Vibrio vulnificus septicemia with shock. Japanese Journal of Medicine, 29, 313-319. doi:10.2169/internalmedicine1962.29.313

[10] Nakafusa, J., Misago, N., Miura, Y., Kayaba, M., Tanaka, T. and Narisawa, Y. (2001) The importance of serum creatine phosphokinase level in the early diagnosis, and as a prognostic factor, of Vibrio vulnificus infection. The British Journal of Dermatology, 145, 280-228. doi:10.1046/j.1365-2133.2001.04347.x

[11] Tajiri, T., Tate, G., Akita, H., Ohike, N., Masunaga, A., Kunimura, T., Mitsuya, T. and Morohoshi, T. (2008) Autopsy cases of fulminant-type bacterial infection with necrotizing fasciitis: Group A (beta) hemolytic Streptococcus pyogenes versus Vibrio vulnificus infection. Pathology International, 58, 196-202. doi:10.1111/j.1440-1827.2007.02211.x

[12] Inoue, H. (2006) Vibrio vulnificus infection of the hand. Journal of Orthopaedic Science, 11, 85-87. doi:10.1007/s00776-005-0965-X

[13] Ikeda, T., Kanehara, S., Ohtani, T. and Furukawa, F. (2006) Endotoxin shock due to Vibrio vulnificus infection. European Journal Dermatology, 16, 423-427.

[14] Chen, Y., Satoh, T., Tokunaga, O. (2002) Vibrio vulnificus infection in patients with liver disease: Report of five autopsy cases. Virchows Archiv, 441, 88-92. doi:10.1007/s00428-002-0613-1

[15] The Ministry of Health, Labour and Welfare of Japan (2008) http://www.mhlw.go.jp/houdou/2008/12/d1/h1225-5d.pdf

[16] Davies, H.D., McGeer, A., Schwartz, B., Green, K., Cann, D., Simor, A.E. and Low, D.E. (1996) Invasive group A streptococcal infections in Ontario, Canada. Ontario group A streptococcal study group. The New England Journal of Medicine, 335, 547-554. doi:10.1056/NEJM199608223350803

[17] Blake, P.A., Merson, M.H., Weaver, R.E., Hollis, D.G. and Heublein, P.C. (1979) Disease caused by a marine Vibrio. Clinical characteristics and epidemiology. The New England Journal of Medicine, 300, 1-5. doi:10.1056/NEJM197901043000101 